\title{
USO DE VORICONAZOL TÓPICO EN QUERATITIS POR ALTERNARIA
}

\section{USE OF TOPICAL VORICONAZOLE IN ALTERNARIA KERATITIS}

\author{
DE-DOMINGO-BARÓN B ${ }^{1}$, RODRÍGUEZ-ARES T², TOURIÑO-PERALBA R ${ }^{2}$, \\ PARDO-SÁNCHEZ F ${ }^{3}$, ROMERO-JUNG P ${ }^{4}$, BARCIA M ${ }^{5}$
}

\section{RESUMEN}

Caso clínico: Hombre de 38 años remitido de otro centro por presentar queratitis fúngica por Alternaria 25 días después de realizarle una queratoplastia penetrante postraumática en ojo derecho. Se instaura tratamiento con voriconazol tópico y oral con buena evolución posterior.

Discusión: Las queratitis fúngicas por Alternaria son poco frecuentes. Su tratamiento es difícil porque la evolución clínica no se correlaciona con la susceptibilidad in vitro del hongo. Producen cuadros clínicos que requieren un rápido diagnóstico y tratamiento, para evitar la pérdida de visión. La combinación de voriconazol tópico y sistémico puede ser una buena alternativa en caso de hongos resistentes al tratamiento convencional.

Palabras clave: Queratitis fúngicas, queratitis resistentes, voriconazol tópico, Alternaria, queratoplastia penetrante.

\section{ABSTRACT}

Clinical case: A 38-year-old man with fungal Alternaria keratitis was referred from another hospital 25 days after post-traumatic penetrating keratoplastia surgery on his right eye. We commenced treatment with topical voriconazole and the condition resolved.

Discussion: Fungal Alternaria keratitis is rare, and treatment is difficult because the clinical response does not correlate well with the antibiotic in vitro sensitivity of the fungus. Clinical cases need to be diagnosed and treated quickly if visual loss is to be avoided. The combination of topical and systemic voriconazole has been shown to be an effective treatment for this condition (Arch Soc Esp Oftalmol 2008; 83: 493-496).

Key words: Fungal keratitis, resistent keratitis, topical voriconazole, Alternaria, penetrating keratoplastia.

\footnotetext{
Recibido: 23/1/07. Aceptado: 16/6/08.

Complejo Hospitalario Universitario de Santiago de Compostela. Santiago de Compostela. España.

1 Licenciada en Medicina. Servicio de Oftalmología.

2 Doctora en Medicina. Servicio de Oftalmología.

3 Doctora en Farmacia. Servicio de Microbiología.

${ }^{4}$ Licenciada en Medicina. Servicio de Microbiología.

5 Licenciado en Farmacología. Servicio de Farmacología.

Correspondencia:

Begoña de Domingo Barón

Moas de Abaixo, 4C

15897 Santiago de Compostela (La Coruña)

España

E-mail: bdedbar@yahoo.es
} 


\section{INTRODUCCIÓN}

Las queratitis fúngicas constituyen una causa rara de infección corneal en países industrializados, siendo un problema importante de salud pública en países tropicales donde la mayoría de la población se ubica en el ámbito rural.

Existe un predominio de las distintas especies en función de la ubicación geográfica. Los hongos filamentosos son típicos de los trópicos, mientras que las levaduras están más relacionadas con factores de riesgo externos.

$\mathrm{Su}$ importancia oftalmológica radica en las dificultades diagnósticas y terapéuticas, que ensombrecen el pronóstico de estos pacientes. Además, su incidencia en los países de clima templado ha aumentado por el uso y abuso de antibióticos y corticoides tópicos, aumento de cirugías corneales, o viajes a países tropicales. La aparición de estas queratitis está favorecida por un fallo en los mecanismos de defensa a nivel corneal, ya sea por una inmunosupresión sistémica o local.

La virulencia de un hongo puede ser debida a su capacidad para resistir a las defensas del huésped, penetrar por la membrana de descemet intacta y provocar necrosis tisular a través de la producción de enzimas, contribuyendo la reacción inflamatoria del huésped al daño tisular.

Estos hechos justifican el interés del oftalmólogo por el estudio de factores predisponentes y características clínicas que faciliten un rápido diagnóstico y la búsqueda de nuevas alternativas terapeúticas más eficaces y menos cruentas.

\section{CASO CLÍNICO}

Paciente de 38 años, con antecedentes de hepatitis, enviado desde la consulta de urgencias a la Unidad de Segmento Anterior de nuestro hospital por sospecha de queratitis infecciosa en ojo derecho. Al paciente se la había practicado en otro centro una queratoplastia penetrante postraumática en dicho ojo 25 días antes. A la exploración oftalmológica presentaba un defecto epitelial central del injerto con edema estromal anterior. Se tomaron muestras para cultivo microbiológico mediante raspado comeal y se instauró tratamiento con voriconazol oral (6 mg/kg cada 12 horas) y antibioticoterapia tópica. Se redujeron los corticoides ante la sospecha de una infección fúngica. El cultivo mostró creci- miento de Alternaria, y se añadió a su tratamiento colirio de voriconazol al $1 \%$ con frecuencia horaria, observándose una disminución clara de la inflamación anterior (fig. 1).

Al cabo de tres meses se le realizó una nueva queratoplastia con inyección intracamerular de Anfotericina B, con buena evolución postoperatoria (fig. 2).

\section{DISCUSIÓN}

Los hongos son organismos eucariotas que no pueden penetrar en un epitelio comeal íntegro. Las especies del género Alternaria producen úlceras comeales de evolución lenta (1), teniendo la mayoría de ellas un origen rural y habiendo recibido en la mayoría de los casos antibióticos y corticoides antes de llegar al oftalmólogo.

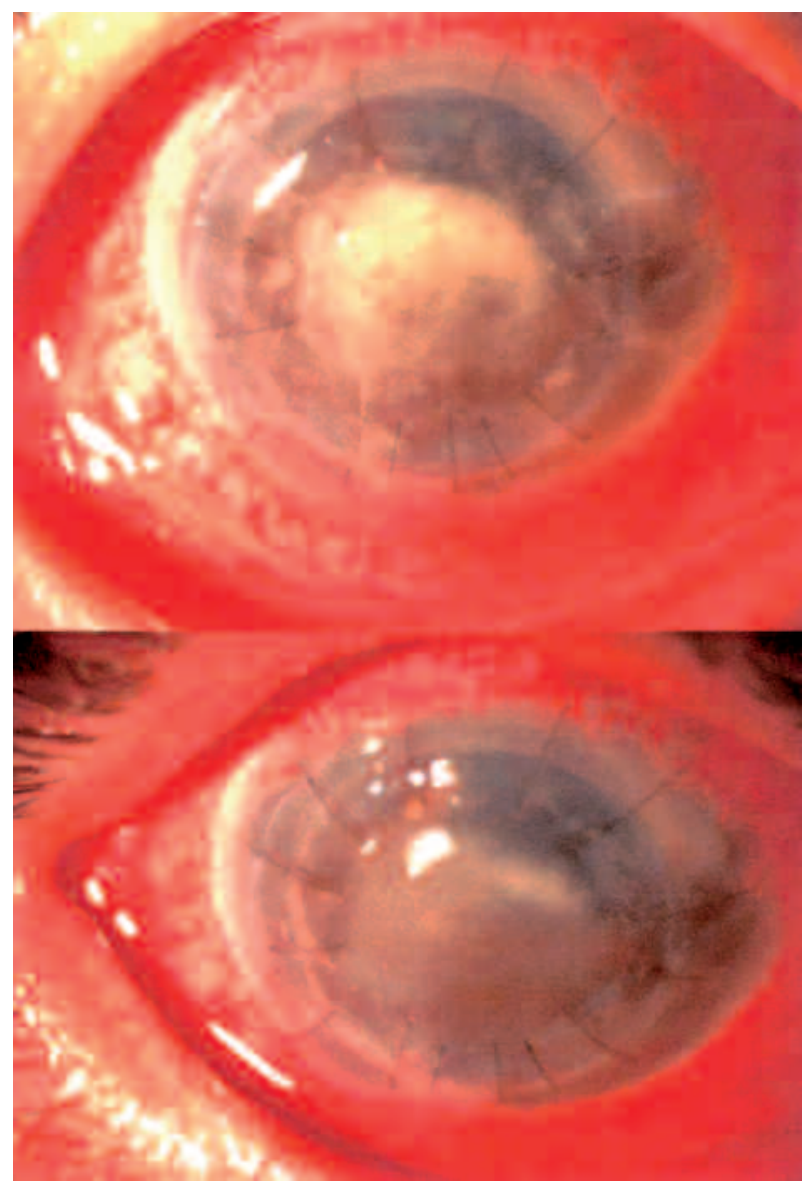

Fig. 1: Evolución de la queratitis por Alternaria desde el inicio del tratamiento con voriconazol tópico hasta pasados cinco días. 


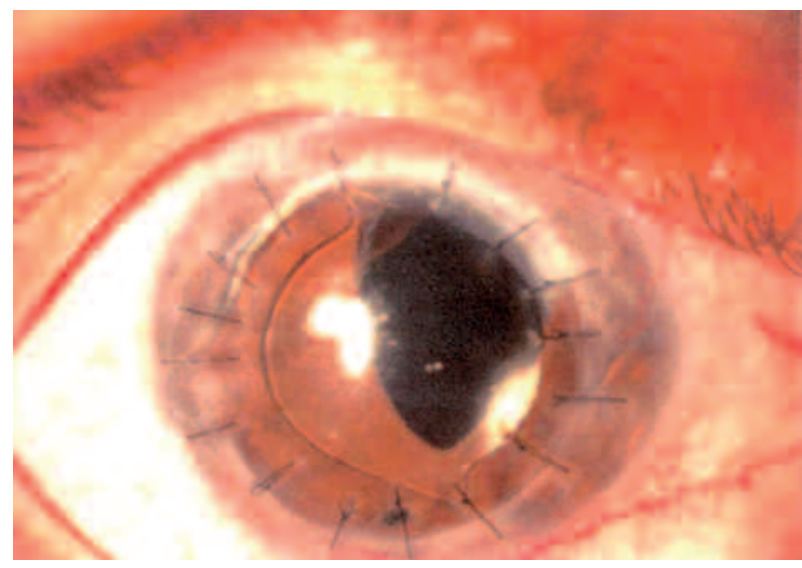

Fig. 2: Aspecto del ojo tres meses tras queratoplastia.

En diversos estudios, el traumatismo es el factor de riesgo más frecuente, seguido por enfermedad sistémica y cirugía local previa. Los traumatismos con vegetales son responsables del $60,5 \%$ de los traumatismos en general.

Nuestro caso se trata de un paciente jóven que tras un traumatismo perforante requiere de la realización de una queratoplastia, a raíz de la cual se observa la aparición de una queratitis fúngica. Una de las complicaciones más serias de las queratoplastias penetrantes son las infecciones oculares postquirúrgicas. La contaminación por hongos del botón donante puede ser la responsable de la aparición de infecciones posteriores. Esto podría explicar la aparición de queratitis fúngica en nuestro paciente. En los modelos experimentales, la inmunosupresión de los tejidos oculares inducida mediante la administración sistémica de metilprednisolona, conduce a un incremento de la severidad de la infección y a una persistencia más prolongada del hongo en la córnea (2). Estudios previos han demostrado la eficacia del voriconazol en el tratamiento de las queratitis fúngicas $(3,4)$. En nuestro caso se utilizó voriconazol tópico al $1 \%$ y sistémico, evidenciándose una mejoría significativa en la inflamación corneal. El voriconazol es un derivado sintético del fluconazol. Su espectro es muy amplio englobando tanto a levaduras como a hongos filamentosos. Se puede emplear vía sistémica (oral/intravenosa) o de forma tópica al 1\%, como fármaco de uso compasivo. Como efectos adversos se han observado un aumento de la fotosensibilidad y enrojecimiento facial tras un mes de tratamiento (5).

Es necesario realizar más estudios, pero parece ser que la combinación de voriconazol tópico y sistémico es una buena alternativa en aquellos casos de queratitis fúngicas resistentes a tratamiento convencional.

\section{BIBLIOGRAFÍA}

1. Ozbek Z, Kang S, Sivalingarn J, Rapuano CJ, Cohen EJ, Harnrnersrnith KM. Voriconazole in the management of alternaria keratitis. Cornea 2006; 25: 242-244.

2. Polizzi A, Siniscalchi C, Mastrornarino A, Sacca SC. Effect of voriconazole on a corneal abscess caused by fusariurn. Acta Ophthalrnol Scand 2004; 82: 762-764.

3. Klont RR, Eggink CA, Rijs AJ, Wesseling P, Verweij PE. Successful treatment of fusariurn keratitis with cornea transplantation and topical and systemic voriconazole. Clin Infect Dis 2005; 40: ell0-e112.

4. Sponsel W, Chen N, Dang D, Paris G, Graybill J, Najvar $L K$, et al. Topical voriconazole as a novel treatment for fungal keratitis. Antirnicrob Agents Chemother 2006; 50: 262-268.

5. Rondeau N, Bourcier T, Chaurneil C, Boredeire V, Touzeau $O$, Scat Y, et al. Fungal keratitis at the Centre Hospitalier National d'Ophtalrnologie des Quinze-Vingts: retrospective study of 19 cases. J Fr Ophtalmol 2002; 25: 890-896. 